TRANSACTIONS OF THE

AMERICAN MATHEMATICAL SOCIETY

Volume 362, Number 8, August 2010, Pages 3947-3962

S 0002-9947(10)05109-3

Article electronically published on March 9, 2010

\title{
CALIBRATIONS ASSOCIATED TO MONGE-AMPÈRE EQUATIONS
}

\author{
MICAH WARREN
}

\begin{abstract}
We show the volume maximizing property of the special Lagrangian submanifolds of a pseudo-Euclidean space. These special Lagrangian submanifolds arise locally as gradient graphs of solutions to MongeAmpère equations.
\end{abstract}

\section{INTRODUCTION}

In this note we discuss calibrations on $\mathbb{R}^{n} \times \mathbb{R}^{n}$, with respect to the pseudoEuclidean metric $d x d y$. The metric $d x d y$ can be expressed explicitly as the indefinite form

$$
d x d y=\frac{1}{2} \sum_{i}\left(d x_{i} \otimes d y_{i}+d y_{i} \otimes d x_{i}\right) .
$$

Hitchin showed how the metric arises naturally in the study of the moduli space of calibrated submanifolds [6. Recently, Kim and McCann [9] have shown that this metric arises naturally in the study of optimal transportation. Our main result is the following 1

Theorem 1.1. Let $\Gamma=(x, \nabla u(x)) \subset \mathbb{R}^{n} \times \mathbb{R}^{n}$ be the gradient graph of a convex function $u \in C^{2}(\Omega)$, for $\Omega$ a bounded, simply connected region with $C^{1}$ connected boundary. If u satisfies the Monge-Ampère equation

$$
\operatorname{det}\left(D^{2} u\right)=c,
$$

then $\Gamma$ is volume maximizing in the pseudo-Euclidean space $\left(\mathbb{R}_{x}^{n} \times \mathbb{R}_{y}^{n}, d x d y\right)$. Precisely, for any homologous, $C^{1}$, space-like, oriented $n$-surface $\Sigma$, with $\partial \Sigma=\partial \Gamma=$ $\Gamma \cap\{x \in \partial \Omega\}$,

$$
\operatorname{Vol}(\Sigma) \leq \operatorname{Vol}(\Gamma)
$$

with equality only if $\Sigma=\Gamma$.

Received by the editors July 17, 2006.

2000 Mathematics Subject Classification. Primary 35J60.

${ }^{1}$ The author recently learned from Reese Harvey that the study of volume maximizing submanifolds was pioneered by Jack Mealy in his dissertation, see [1]. Mealy proved Theorem [1.1 for a special Lagrangian equation describing the submanifolds in a different coordinate system.

(C)2010 American Mathematical Society
ublic domain 28 years from publication

Reverts to public domain 28 years from publication 3947 
We also show (section 6) that the Monge-Ampère equations (1.1)

$$
\sum \ln \lambda_{i}=\ln c
$$

(where $\lambda_{i}$ are the eigenvalues of $D^{2} u$ ) are part of a one parameter family of special Lagrangian equations which includes the special Lagrangian equations of Harvey and Lawson [5]:

$$
\sum_{i} \arctan \lambda_{i}=c .
$$

A Lagrangian submanifold of $\mathbb{R}^{n} \times \mathbb{R}^{n}$ is one that can be described locally as a gradient graph, $(x, \nabla u(x))$. Hitchin $([6, \S 5)$ introduced a definition of "special Lagrangian" for Lagrangian submanifolds of $\left(\mathbb{R}^{n} \times \mathbb{R}^{n}, d x d y\right)$ and demonstrated that a gradient graph $(x, \nabla u(x))$ is special precisely when the potential $u(x)$ satisfies (1.1). Jost and Xin $([8, \S 4)$ then showed that such a submanifold has vanishing mean curvature. Yu Yuan observed that (1.1) belongs to a larger family of special Lagrangian equations and suggested that it is possible to find a calibration associated to (1.1). This is what we show now. Specifically, we show that if $u(x)$ is a solution to (1.1), then the gradient graph $(x, \nabla u(x))$ is a calibrated submanifold of $\left(\mathbb{R}^{n} \times \mathbb{R}^{n}, d x d y\right)$, and is consequently volume maximizing. In fact, any submanifold which is locally described by gradient graphs of functions satisfying (1.1) is calibrated, and therefore volume maximizing. Theorem 1.1 can be stated so as to allow a slightly larger class of $n$-surfaces, as is shown in section 3 .

The results here are generalized in [10], where we see that it is possible to use the language of currents to formulate a more general result than above, which reads more like the analogous theorem for Riemannian geometry ([5], Theorem 4.2, p. 59). The arguments in [10] are based on a definition of mass in pseudo-Euclidean space which severely penalizes submanifolds (or more generally, $n$-currents) which fail to be space-like. Here we will use a more naïve definition of volume, and because of this we will require certain conditions on the domain and on competing $n$-surfaces.

The study of calibrated Lagrangian submanifolds of $\mathbb{R}^{n} \times \mathbb{R}^{n}$ began with the work of Harvey and Lawson $\left([5, \S 3)\right.$, who studied Lagrangian submanifolds of $\mathbb{C}^{n} \cong$ $\mathbb{R}^{n} \times \mathbb{R}^{n}$ with the Euclidean metric $\delta_{0}$, and showed that a Lagrangian submanifold is calibrated, and therefore volume minimizing, if and only if the potential $u(x)$ is a solution to the special Lagrangian equation (1.2).

McLean ([12, Theorem 3.6, 3.10) showed that given $\Sigma \subset M$ a compact special Lagrangian submanifold, the moduli space $X$ of special Lagrangian manifolds near $\Sigma$ in $M$ is itself a manifold which carries a natural Riemannian metric. Hitchin ([6], Proposition 2) showed that this metric can be obtained locally by embedding the moduli space $X$ into $\left(V \oplus V^{*}, d x d y\right)$, where $V=H^{1}(\Sigma, \mathbb{R})$. Hitchin then showed ([6], Proposition 3) that $X$ is a special Lagrangian submanifold of $\left(V \oplus V^{*}, d x d y\right)$, and that special Lagrangian submanifolds arise as solutions to the Monge-Ampère equation, $\operatorname{det}\left(D^{2} u\right)=c$.

In section 2, we summarize the argument of Harvey and Lawson. In section 3, we show the linear algebra necessary for the calibration argument and then prove the main result. We follow with some counterexamples to show how the theory breaks down without certain conditions, and in section 5 we discuss the calibrations in terms of homogeneous spaces. Section 6 discusses the family of nonlinear equations which connect the equations (1.1) to (1.2), and we conclude with a nongraphical example. 


\section{Preliminaries}

For $\Gamma$ the graph of $F$ over a bounded region $\Omega \subset \mathbb{R}^{n}$, the volume of $\Gamma$ is given by the functional

$$
\operatorname{Vol}(\Gamma)=\int_{\Omega} \sqrt{\operatorname{det}\left(I+(D F)(D F)^{T}\right)} d x
$$

when the metric on $\mathbb{R}^{n} \times \mathbb{R}^{n}$ is the Euclidean metric $\delta_{0}$. When the ambient metric is the pseudo-Euclidean metric $g_{0}$, the volume functional is given by

$$
\operatorname{Vol}(\Gamma)=\int_{\Omega} \sqrt{\operatorname{det}\left(D F+(D F)^{T}\right)} d x .
$$

This definition requires that $\operatorname{det}\left(D F+(D F)^{T}\right)$ is positive, which will be the case when $u$ is convex. See [10] for a more general definition.

We may use the idea of a calibration to detect maximal surfaces in the pseudoRiemannian setting, provided we pay careful attention to orientation as it arises. Given an oriented $k$-plane $\xi$ and a $k$-form $\varphi$, we will say that $\xi$ is oriented with respect to $\varphi$ whenever $\varphi(\xi)>0$. Let $M$ be a pseudo-Riemannian manifold with index $n-k$, and suppose $\varphi$ is a closed exterior $k$-form on $M$ with the property that on all space-like $k$-planes oriented with respect to $\varphi$,

$$
\varphi(\xi) \geq \operatorname{Vol}(\xi)
$$

Such a $\varphi$ is called a calibration. Now let $\Sigma$ be any compact, oriented, $k$-dimensional, space-like submanifold with the property that $\left.\varphi\right|_{\Sigma}(\xi)=\left.V o l\right|_{\Sigma}(\xi)$ for all oriented (with respect to the orientation on $\Sigma$ ) tangent $k$-planes $\xi$. If $\Sigma$ is homologous to $\Sigma^{\prime}$, then $\Sigma^{\prime}$ has a prescribed orientation, and it is with respect to this orientation that we apply Stokes's Theorem and obtain

$$
\int_{\Sigma} d V o l=\int_{\Sigma} \varphi=\int_{\Sigma^{\prime}} \varphi \geq \int_{\Sigma^{\prime}} d V o l
$$

as long as the oriented tangent planes of $\Sigma^{\prime}$ are $\varphi$-oriented. It is possible, however, for the orientation of $\Sigma^{\prime}$ to produce tangent planes which are not oriented with respect to $\varphi$. If this disagreement occurs, the calibrating inequality is reversed, and we are unable to make the volume comparison. This can happen if $\Sigma^{\prime}$ is disconnected or is singular along a significant subset. The conditions in Theorem 1.1 are sufficient to preclude any such pathology, as we will see in section 3 .

For the Euclidean case, Harvey and Lawson $([5], \S 3)$ define the family of forms

$$
\alpha_{\theta}=\operatorname{Re}\left(e^{-\sqrt{-1} \theta} d z\right)
$$

where $d z=d z_{1} \wedge \ldots \wedge d z_{n}$, and show that these are calibrations on $\mathbb{C}^{n}$. We briefly recall the idea.

For any real $n$-plane $\xi$ in $\mathbb{R}^{n} \times \mathbb{R}^{n}=\mathbb{C}^{n}$, we choose an oriented orthonormal basis $v_{1}, \ldots, v_{n}$. Now $v_{i} \in \mathbb{R}^{n} \times \mathbb{R}^{n}$, but we will identify these with their corresponding complex vectors and consider $v_{i} \in \mathbb{C}^{n}$. Define a complex-linear map $A: \mathbb{C}^{n} \rightarrow \mathbb{C}^{n}$ by

$$
\begin{gathered}
A\left(e_{x_{i}}\right)=v_{i}, \\
A\left(e_{y_{i}}\right)=J v_{i},
\end{gathered}
$$

which is represented by a complex-valued matrix $A \in M(n, \mathbb{C})$, namely, the complex $n \times n$ matrix with columns $v_{1}, \ldots, v_{n}$. This also defines a real-linear map 
$\hat{A}: \mathbb{R}^{n} \times \mathbb{R}^{n} \rightarrow \mathbb{R}^{n} \times \mathbb{R}^{n}$, represented by a real-valued matrix $\hat{A} \in M(2 n, \mathbb{R})$. Now

$$
|\xi \wedge J \xi|=\operatorname{det}_{\mathbb{R}} \hat{A}=\left|\operatorname{det}_{\mathbb{C}} A\right|^{2}=|d z(\xi)|^{2}=\alpha^{2}(\xi)+\beta^{2}(\xi),
$$

where $\alpha(\xi)=\operatorname{Re}(d z(\xi))$ and $\beta(\xi)=\operatorname{Im}(d z(\xi))$. By Hadamard's Inequality, $|\xi|^{2} \geq$ $|\xi \wedge J \xi|$, with equality if and only if $\xi$ is Lagrangian. So now we have

$$
|\xi|^{2} \geq|\xi \wedge J \xi|=\alpha^{2}(\xi)+\beta^{2}(\xi) \geq \alpha^{2}(\xi),
$$

with equality if and only if $\xi$ is Lagrangian and $\beta(\xi)=0$.

If $\xi$ is Lagrangian, then $v_{1}, \ldots, v_{n}$ is an orthonormal basis for $\mathbb{C}^{n}$, so $A \in U(n)$. With the action of $S O(n)$ on $U(n)$ given by $n \times n$ matrix multiplication, choosing an orthonormal basis associates to each Lagrangian plane $\xi$ an $A \in U(n)$ which is unique up to a factor of $S O(n)$. With this association, we have a transitive action of $U(n)$ on the set of Lagrangian planes (which we call $L$ ). The isotropy group of $\xi_{0}=\mathbb{R}_{x}^{n}$ is $S O(n)$, so $L$ is a homogeneous space and the complex determinant descends to a map

$$
\Theta: L \approx U(n) / S O(n) \stackrel{\text { detc }}{\rightarrow} S^{1} .
$$

For any $\xi \in L, d z(\xi)=e^{\sqrt{-1} \Theta(\xi)}$. Taking

$$
\alpha_{\theta}=\operatorname{Re}\left(e^{-\sqrt{-1} \theta} d z\right),
$$

we see that $\alpha_{\theta}(\xi)=1$ if and only if $\Theta(\xi)=\theta$; thus the $\alpha_{\theta}$ 's are calibrations for $\mathbb{C}^{n}$.

The special Lagrangian equations may then be deduced from the condition

$$
\arg \left(\operatorname{det}\left(I+\sqrt{-1} D^{2} u\right)\right)=\arg \left(\left(1+\sqrt{-1} \lambda_{1}\right) \ldots\left(1+\sqrt{-1} \lambda_{n}\right)\right)=c
$$

for some fixed $c$. This equation is satisfied precisely when $\Theta$ is constant along the graph of $\nabla u$.

\section{Calibrations For PSEUdo-Euclidean SPACE}

In the pseudo-Euclidean setting, a Lagrangian submanifold $\Sigma$ is called special $\left([6]\right.$, p. 510) if a linear combination of the volume forms $d x_{1} \wedge \ldots \wedge d x_{n}$ and $d y_{1} \wedge$ $\ldots \wedge d y_{n}$ vanish along $\Sigma$.

Proposition 3.1. For $c>0$,

$$
\Phi_{c}=\frac{1}{2}\left[c d x_{1} \wedge \ldots \wedge d x_{n}+\frac{1}{c} d y_{1} \wedge \ldots \wedge d y_{n}\right]
$$

is a calibration for $\left(\mathbb{R}^{n} \times \mathbb{R}^{n}, d x d y\right)$. Suppose $\xi$ is an oriented space-like $n$-plane in $\mathbb{R}^{n} \times \mathbb{R}^{n}$ with $\Phi_{c}(\xi)>0$. Then

$$
\Phi_{c}(\xi) \geq \operatorname{Vol}(\xi)
$$

with equality if and only if $\xi$ is special Lagrangian, that is, if

$$
d y_{1} \wedge \ldots \wedge d y_{n}(\xi)=c^{2} d x_{1} \wedge \ldots \wedge d x_{n}(\xi) .
$$

In the development of the special Lagrangian calibrations, Harvey and Lawson used Hadamard's Inequality to compare Lagrangian planes to non-Lagrangian planes. In order to prove the Proposition 3.1, we need a result which serves this purpose in the pseudo-Euclidean case. The following lemma is from linear algebra. 
Lemma 3.1. Suppose $Q \in G L(n, \mathbb{R})$ satisfies $Q_{i j} x^{i} x^{j}>0$, for all $0 \neq x \in \mathbb{R}^{n}$. Then

$$
\operatorname{det}(Q) \geq \operatorname{det}\left(\frac{Q+Q^{T}}{2}\right)
$$

with equality if and only if $Q=Q^{T}$.

Proof of Lemma 3.1. The matrix $Q$ can be written $Q=S+A$, with $S$ symmetric and $A$ antisymmetric. Choose a basis so that $S$ is a diagonal matrix. With respect to this basis,

$$
Q=\left(\begin{array}{cccc}
\lambda_{1} & a_{12} & \ldots & a_{1 n} \\
-a_{12} & \lambda_{2} & \ldots & a_{2 n} \\
\ldots & \ldots & \ldots & \ldots \\
-a_{1 n} & -a_{2 n} & \ldots & \lambda_{n}
\end{array}\right)
$$

Expand the determinant of $\mathrm{Q}$, and group the terms according to the number of $\lambda_{i}$ 's each terms contains. For all $k \leq n$ define

$$
P_{k}=\text { the sum of all terms containing exactly } k \lambda_{i} \text { 's. }
$$

We see that $P_{n}$ consists of one term, namely $\lambda_{1} \ldots \lambda_{n}=\sigma_{n}(S)$, and that there are no terms with $(n-1) \lambda_{i}$ 's, so $P_{n-1}=0$. For $P_{n-k}, k \geq 2$, we fix $i_{1}, \ldots, i_{n-k}$, and look at the terms containing $\lambda_{i_{1}} \ldots \lambda_{i_{n-k}}$. These occur as the determinant of a matrix, which after an orthogonal change of basis, looks like

$$
\left(\begin{array}{cccccc}
\lambda_{i_{1}} & & & & & \\
& \ldots & & & & \\
& & \lambda_{i_{n-k}} & & & \\
& & & 0 & a_{j_{1} j_{2}} & \ldots \\
& & & -a_{j_{1} j_{2}} & 0 & \\
& & & \cdots & &
\end{array}\right)
$$

with $j_{1}, \ldots, j_{k} \notin\left\{i_{1}, \ldots, i_{n-k}\right\}$. This determinant is the product of the determinants of a positive diagonal matrix and an antisymmetric matrix. It follows that $P_{n-k} \geq$ 0 . We also see that

$$
P_{n-2}=\sum_{i<j} a_{i j}^{2} \lambda_{1} \ldots \hat{\lambda}_{i} \hat{\lambda}_{j} \ldots \lambda_{n}
$$

which is strictly positive unless $a_{i j}=0$, for all $i, j$. We conclude that

$$
\operatorname{det}(Q)=P_{n}+P_{n-2}+\ldots+P_{0} \geq P_{n}=\operatorname{det}(S)=\operatorname{det}\left(\frac{Q+Q^{T}}{2}\right)
$$

with equality if and only if $Q=Q^{T}$.

Proof of Proposition 3.1. If $\xi$ is a space-like tangent plane, the projection onto $\mathbb{R}_{x}^{n}$ is full rank, and we can take a basis for $\xi$ of the form

$$
v_{i}=e_{x_{i}}+w_{i}^{j} e_{y_{j}}, \quad i=1, \ldots, n .
$$

Let $Q$ be the matrix given by $Q_{i j}=w_{i}{ }^{j}$. If $g$ is the induced metric, then with respect to this frame, the tensor $2 g_{i j}=2 g\left(v_{i}, v_{j}\right)$ becomes $\left(Q+Q^{T}\right)_{i j}$, so

$$
\operatorname{Vol}\left(v_{1} \wedge \ldots \wedge v_{n}\right)=\sqrt{\operatorname{det}\left(\frac{Q+Q^{T}}{2}\right)} .
$$


Now

$$
\Phi_{c}\left(v_{1} \wedge \ldots \wedge v_{n}\right)=\frac{1}{2}\left[c+\frac{1}{c} \operatorname{det}(Q)\right] \geq \sqrt{\operatorname{det} Q}
$$

with equality if and only if $\operatorname{det}(Q)=c^{2}$, so

$$
\Phi_{c}\left(v_{1} \wedge \ldots \wedge v_{n}\right) \geq \sqrt{\operatorname{det}(Q)} .
$$

From the above lemma

$$
\sqrt{\operatorname{det}(Q)} \geq \sqrt{\operatorname{det}\left(\frac{Q+Q^{T}}{2}\right)}=\operatorname{Vol}\left(v_{1} \wedge \ldots \wedge v_{n}\right)
$$

with equality only if $\mathrm{Q}$ is symmetric, that is, if $\xi$ is Lagrangian. Hence

$$
\Phi_{c}\left(v_{1} \wedge \ldots \wedge v_{n}\right) \geq \operatorname{Vol}\left(v_{1} \wedge \ldots \wedge v_{n}\right)
$$

with equality if and only if $\operatorname{det}(Q)=c^{2}$ and $Q=Q^{T}$.

We now prove our main result.

Theorem 3.2. Suppose $\Omega \subset \mathbb{R}^{n}$ is a bounded region, and $u \in C^{2}(\Omega)$ is a convex solution to the Monge-Ampère equation

$$
\operatorname{det}\left(D^{2} u\right)=c^{2} .
$$

Then in the pseudo-Euclidean space $\left(\mathbb{R}_{x}^{n} \times \mathbb{R}_{y}^{n}, d x d y\right)$, the gradient graph of $u(x)$, $\Gamma=(x, \nabla u(x))$, is volume maximizing in the following sense:

Let $\Sigma$ be an oriented $n$-surface which is homologous to $\Gamma$ in $\mathbb{R}^{n} \times \mathbb{R}^{n}$, with $\partial \Sigma=$ $\partial \Gamma=\Gamma \cap\{x \in \partial \Omega\}$. If any of the following hold

- $\partial \Omega$ is connected and $\Sigma$ is $C^{1}$ and space-like,

- $\Sigma$ is connected, $C^{1}$ and space-like, or

- $\Sigma$ is $C^{1}$ and space-like except on a set $\Sigma_{0}$, which has $n$-dimensional Hausdorff measure zero, such that $\Sigma \backslash \Sigma_{0}$ is connected,

then $\operatorname{Vol}(\Gamma) \geq \operatorname{Vol}(\Sigma)$. If $\Omega$ is simply connected with $C^{1}$ connected boundary, and $\Sigma$ is $C^{1}$, then the equality holds only if $\Sigma=\Gamma$.

Proof of Theorem 3.2 and Theorem 1.1. Suppose $u \in C^{2}(\Omega)$ with $\operatorname{det}\left(D^{2} u\right)=c^{2}$ on $\Omega$. Then the coordinates in $\mathbb{R}^{n}$ give us the tangent frame

$$
\partial_{i}=e_{x_{i}}+\sum_{j} u_{i j} e_{y_{j}}
$$

and we compute

$$
\begin{aligned}
\Phi_{c}\left(\partial_{1} \wedge \ldots \wedge \partial_{n}\right) & =\frac{1}{2}\left[c+\frac{1}{c} c^{2}\right]=c, \\
\operatorname{Vol}\left(\partial_{1} \wedge \ldots \wedge \partial_{n}\right) & =\sqrt{\operatorname{det}\left(D^{2} u\right)}=c .
\end{aligned}
$$

So

$$
\left.\operatorname{Vol}\right|_{\Gamma}=\left.\Phi_{c}\right|_{\Gamma}
$$

and $\Gamma$ is a calibrated submanifold. For any homologous $n$-surface $\Sigma$, we know that

$$
\int_{\Sigma} \Phi_{c}=\int_{\Gamma} \Phi_{c}=\operatorname{Vol}(\Gamma)
$$

so our task is to show that each of the listed conditions implies that the oriented planes for $\Sigma$ are oriented with respect to $\Phi_{c}$, giving

$$
\left.\Phi_{c}\right|_{\Sigma} \geq\left.\operatorname{Vol}\right|_{\Sigma}
$$


We begin by showing that the first condition implies the second condition. Regarded as a linear map, $g_{0} \in G L(2 n, \mathbb{R})$ has two eigenvalues, +1 and -1 , so we can decompose $\mathbb{R}^{2 n}$ into the eigenspaces $E_{-1}$ and $E_{1}$ corresponding to these two eigenvalues. Let $\operatorname{Proj}_{1}$ be the projection of $\mathbb{R}^{n} \times \mathbb{R}^{n}$ onto $E_{1}$. If $\Sigma$ is $C^{1}$ and space-like, the projection $\left.\operatorname{Proj}_{1}\right|_{\Sigma} \rightarrow E_{1}$ must be full rank, so it must be an open map. It follows that each component of $\Sigma$ must have nonempty boundary. Since $\partial \Sigma=\partial \Gamma$, $\partial \Sigma$ must be connected. Each component of $\Sigma$ intersects $\partial \Sigma$, so $\Sigma$ consists of a single component.

It is clear that the second condition implies the third. So now assume $\Sigma$ is $C^{1}$ and space-like except on a set $\Sigma_{0}$, which has $n$-dimensional Hausdorff measure zero, and $\Sigma \backslash \Sigma_{0}$ is connected. The integral $\int_{\Sigma} \Phi_{c}$ is positive, so for the induced orientation on $\Sigma, \Phi_{c}(\xi)$ is positive for some oriented tangent plane $\xi_{s}=T_{s} \Sigma$ at some point $s \in \Sigma$. Since $V o l$ does not vanish on $\Sigma \backslash \Sigma_{0}$, it follows from the $C^{1}$ assumption that $\Phi_{c}\left(\xi_{s}\right) \geq \operatorname{Vol}\left(\xi_{s}\right)>0$ for all $s \in \Sigma \backslash \Sigma_{0}$. Hence

$$
\operatorname{Vol}(\Sigma)=\int_{\Sigma} d \operatorname{Vol} \leq \int_{\Sigma} \Phi_{c}=\operatorname{Vol}(\Gamma) .
$$

Uniqueness. It is clear by Proposition 3.1 that equality will only occur if $\Sigma$ is a special Lagrangian surface, locally described by gradient graphs of $\operatorname{det} D^{2} u=c^{2}$. In order to use the comparison principle, we first show that $\Sigma$ is globally described by a single graph over $\Omega$.

Let $\operatorname{Proj}_{x}$ be the projection of $\mathbb{R}^{n} \times \mathbb{R}^{n}$ onto $\mathbb{R}_{x}^{n}$, and let $\Omega_{\Sigma}=\operatorname{Proj}_{x}(\Sigma)$. We observe that, due to the space-like condition, the projection $\operatorname{Proj}_{x}$ is open on the interior of $\Sigma$, so $\partial \Omega_{\Sigma} \subset \operatorname{Proj}_{x}(\partial \Sigma)=\partial \Omega$.

Let $p=\left(x_{0}, \nabla u\left(x_{0}\right)\right) \in \partial \Omega=\partial \Sigma$, for $x_{0}$ an extreme point of $\Omega$. Because $\partial \Omega_{\Sigma} \subset$ $\partial \Omega$, it follows that $x_{0}$ is also an extreme point for $\Omega_{\Sigma}$, and that the inward pointing normals for $\partial \Omega$ and $\partial \Omega_{\Sigma}$ must agree at $x_{0}$. The regions $\Omega_{\Sigma}$ and $\Omega$ then must intersect on a nontrivial open set near $x_{0}$. Using the openness of $\operatorname{Proj}_{x}$, together with the boundary condition $\partial \Omega_{\Sigma} \subset \partial \Omega$, it is then easy to check that $\Omega_{\Sigma} \cap \Omega$ is relatively closed and relatively open as a subset of $\Omega$; hence $\Omega \subset \Omega_{\Sigma}$.

Take a cover of $\Sigma \backslash \partial \Sigma$ by open sets $\Sigma_{i}$ (open with respect to $\Sigma$ ), where each is a gradient graph $\Sigma_{i}=\left\{\left(x, \nabla v_{i}\right) \mid x \in U_{i}\right\}$, for $v_{i}$ solutions to $\operatorname{det} D^{2} v_{i}=c^{2}$ on $U_{i}$. Let $\hat{U}_{1}=U_{1}$; then recursively define $\hat{U}_{i}=U_{i} \backslash \bigcup_{k<i} \hat{U}_{k}$ and define $\hat{\Sigma}_{i}=\left\{\left(x, \nabla v_{i}\right) \mid x \in\right.$ $\hat{U}_{i}$. The disjoint union $\bigcup_{i} \hat{U}_{i}$ is then an open set that contains $\Omega$, and the disjoint union $\bigcup_{i} \hat{\Sigma}_{i}$ is a subset of $\Sigma$.

Suppose that $\bigcup_{i} \hat{\Sigma}_{i} \neq \Sigma \backslash \partial \Sigma$. Then there exists an open subset $U_{j k} \subset U_{j} \cap U_{k}$, with $j<k$, such that $\Sigma_{j}$ and $\Sigma_{k}$ are both graphs over $U_{j k}$ but are not equal. Then $\Sigma$ must contain the disjoint union

$$
\Sigma^{\prime}=\bigcup_{i}\left\{\left(x, \nabla v_{i}\right) \mid x \in \hat{U}_{i}\right\} \bigcup\left\{\left(x, \nabla v_{k}\right) \mid x \in U_{j k}\right\} .
$$

However, integrating over $\Sigma$ gives

$$
\int_{\Sigma} d V o l \geq \int_{\Sigma^{\prime}} d V o l \geq \int_{\Omega} c d x+\int_{U_{j k}} c d x>c|\Omega|=\operatorname{Vol}(\Gamma),
$$

contradicting the inequality in the conclusion of Theorem 3.2 We conclude that $\Sigma \backslash \partial \Sigma=\bigcup_{i} \hat{\Sigma}_{i}$ and $\operatorname{Proj}_{x}(\Sigma \backslash \partial \Sigma)=\Omega$. The gradients $\nabla v_{j}$ and $\nabla v_{k}$ must agree on all overlaps $U_{j} \cap U_{k}$, so we can extend any of the $\mathbb{R}^{n}$-valued functions $\nabla v_{j}$ to an 
$\mathbb{R}^{n}$-valued function $F$ on all of $\Omega$. We have assumed that $\Omega$ is simply connected, so $F=\nabla v$, for some $v$ satisfying $\operatorname{det} D^{2} v=c^{2}$, on all of $\Omega$. To compare $u$ with $v$ we note that $\nabla u=\nabla v$ is already fixed around the boundary of $\Omega$, which is connected, so we may integrate $u$ and $v$ around the boundary and conclude that $u$ and $v$ differ by a constant. Applying the comparison principle for nonlinear elliptic equations (cf. [4, Theorem 17.1) gives uniqueness.

\section{Counterexamples}

We give some examples to show that the inequality in Theorem 3.2 fails, if we do not assume any of the conditions on $\Sigma$. For small $\epsilon$, let $\Omega \subset \mathbb{R}^{2}$ be the annulus

$$
\left\{x=\left.\left(x_{1}, x_{2}\right)|| x\right|^{2} \in[1,1+\epsilon]\right\}
$$

and let

$$
\Gamma=\left\{\left(x_{1}, x_{2}, x_{1}, x_{2}\right) \mid x \in \Omega\right\} \subset \mathbb{R}^{4}
$$

be the gradient graph of $|x|^{2} / 2$. Let $\Sigma=\Sigma_{1} \cup \Sigma_{2}$, where

$$
\Sigma_{1}=\left\{\left(x_{1}, x_{2}, x_{1}, x_{2}\right) \mid x \in \overline{B_{1}}\right\},
$$

and let

$$
\Sigma_{2}=\left\{\left(x_{1}, x_{2}, x_{1}+\eta(x), x_{2}+\eta(x)\right) \mid x \in \overline{B_{1+\epsilon}}\right\},
$$

where $\eta(x)$ is a small function which is positive on the interior of $B_{1+\epsilon}$ and vanishes on $\partial B_{1+\epsilon}$. With a suitable orientation, the disconnected set $\Sigma$ is homologous to $\Gamma$. We see that $\operatorname{Vol}(\Sigma)$ is very close to $2 \pi$, whereas $\operatorname{Vol}(\Gamma)$ is very close to 0 , as we have chosen $\epsilon$ and $\eta$ small.

To obtain a connected $\Sigma$ for which Theorem 3.2 fails, we alter the previous example slightly. With $\Omega$ as above, define a small "bridge" region

$$
\Omega^{\prime}=\Omega \cap\left\{x_{1}>0\right\} \cap\left\{x_{2} \in[-\epsilon, \epsilon]\right\} .
$$

Let $\Gamma$ be the gradient graph of $|x|^{2} / 2$ over $\Omega \backslash \Omega^{\prime}$, let $\Sigma_{1}$ be the gradient graph of $|x|^{2} / 2$ over $B_{1} \cup \Omega^{\prime}$, and let $\Sigma_{2}$ be as above. Then $\Sigma=\Sigma_{1} \cup \Sigma_{2}$ is connected and homologous to $\Gamma$, and is space-like except on a singular set which is one-dimensional, but nonetheless disconnects $\Sigma$. Again we have $\operatorname{Vol}(\Sigma)$ close to $2 \pi$ and $\operatorname{Vol}(\Gamma)$ close to 0 , so the inequality in Theorem 3.2 does not hold.

\section{From the Viewpoint of Lie groups}

One can also approach the theory of special Lagrangian calibrations for pseudoEulidean space on the level of homogeneous spaces, following the approach of Harvey and Lawson. We use some notation and terminology of $[2]$. Let $g_{0}=2 d x d y$. First, noting that $U(n)=O(2 n) \cap S p(2 n)$, we consider the "para-unitary" group $U^{\pi}=O_{g_{0}}(n, n) \cap S p(2 n)$, where

$$
O_{g_{0}}(n, n)=\left\{A \in M(2 n, \mathbb{R}) \mid(A u, A v)_{g_{0}}=(u, v)_{g_{0}}, \forall u, v \in \mathbb{R}^{n} \times \mathbb{R}^{n}\right\} .
$$

Some standard Lie algebra computations will show that (Proposition 4 of [2])

$$
U^{\pi}=\left\{\left(\begin{array}{cc}
A & 0 \\
0 & B
\end{array}\right) \mid A B^{T}=I \in M(n, \mathbb{R})\right\} .
$$

Next, consider the space $L^{+}$of all space-like Lagrangian planes. Given $\xi \in L^{+}$, choose an oriented basis, $v_{1}, \ldots, v_{n}$, with each $v_{i} \in \mathbb{R}^{n} \times \mathbb{R}^{n}$, which is orthonormal 
with respect to $g_{0}$, and let $(A, B)$ be the $n \times 2 n$ matrix with rows $v_{i}$. Then associate to each $\xi \in L^{+}$the element

$$
\left(\begin{array}{cc}
A & 0 \\
0 & B
\end{array}\right) \in U^{\pi}
$$

which is unique up to a factor of $S O(n)$, where the $S O(n)$ action on $U^{\pi}$ is defined by

$$
S \cdot\left(\begin{array}{cc}
A & 0 \\
0 & B
\end{array}\right)=\left(\begin{array}{cc}
S A & 0 \\
0 & S B
\end{array}\right) .
$$

This gives the set of space-like Lagrangian planes $L^{+}$the structure of a homogeneous space $L^{+} \approx U^{\pi} / S O(n)$. The homomorphism $\Theta^{+}: U^{\pi} \rightarrow O_{g_{0}}(1,1)$ given by

$$
\left(\begin{array}{cc}
A & 0 \\
0 & B
\end{array}\right) \mapsto\left(\begin{array}{cc}
\operatorname{det}(A) & 0 \\
0 & \operatorname{det}(B)
\end{array}\right)
$$

descends to a map $L^{+} \rightarrow O_{g_{0}}(1,1) \approx H^{1}$, where $H^{1}$ is the pseudo-circle $\{(s, t) \mid t>$ $0, s t=1\}$. A special Lagrangian submanifold is one whose tangent planes lie in a single fiber of $\Theta^{+}$. We can see then that the calibrations are

$$
\Phi_{c}(\xi)=\frac{1}{2}\left(c \operatorname{det} A+\frac{1}{c} \operatorname{det} B\right) \geq \operatorname{Vol}(\xi)
$$

and the special Lagrangian equations are $\operatorname{det} D^{2} u=c^{2}>0$, where the value $c^{2}$ is the pseudo-phase analogous to the phase $c$ in section 2 .

\section{A FAMILY OF NONLINEAR EQUATIONS}

By taking linear combinations of the metrics $\delta_{0}$ and $g_{0}=2 d x d y$, we obtain a family of metrics on $\mathbb{R}^{n} \times \mathbb{R}^{n}$ :

$$
g_{t}=\cos t g_{0}+\sin t \delta_{0} .
$$

We find that the extremal Lagrangian surfaces in $\left(\mathbb{R}^{n} \times \mathbb{R}^{n}, g_{t}\right)$ arise as solutions to a family of special Lagrangian equations:

$$
\begin{gathered}
\text { for } t=0 \quad \sum_{i} \ln \lambda_{i}=c, \\
\text { for } t \in\left(0, \frac{\pi}{4}\right) \quad \sum_{i} \ln \left(\frac{\lambda_{i}+a-b}{\lambda_{i}+a+b}\right)=c, \\
\text { for } t=\frac{\pi}{4} \quad \sum_{i} \frac{1}{1+\lambda_{i}}=c, \\
\text { for } t \in\left(\frac{\pi}{4}, \frac{\pi}{2}\right) \quad \sum_{i} \arctan \left(\frac{\lambda_{i}+a-b}{\lambda_{i}+a+b}\right)=c, \\
\text { for } t=\frac{\pi}{2} \quad \sum_{i} \arctan \lambda_{i}=c,
\end{gathered}
$$

where $a=\cot t$ and $b=\sqrt{\left|\cot ^{2} t-1\right|}$. Further, we have the following extremal volume property of special Lagrangian graphs in $\left(\mathbb{R}^{n} \times \mathbb{R}^{n}, g_{t}\right)$.

Theorem 6.1. i) Suppose $u \in C^{2}(\Omega), \Omega \subset \mathbb{R}^{n}$, is a solution to (6.3). If the metric $g_{t}$ restricts to a positive definite metric on $\Gamma=(x, \nabla u(x))$, then $\Gamma$ is volume maximizing among homologous, $C^{1}$, space-like $n$-surfaces in $\left(\mathbb{R}^{n} \times \mathbb{R}^{n}, g_{t}\right)$, as in Theorem 1.1 . 
ii) Suppose $u \in C^{2}(\Omega), \Omega \subset \mathbb{R}^{n}$, is a solution to (6.4). Then the volume of $\Gamma=(x, \nabla u(x))$ is equal to the volume of any homologous, $C^{1}$, space-like $n$-surface in $\left(\mathbb{R}^{n} \times \mathbb{R}^{n}, g_{\frac{\pi}{4}}\right)$.

iii) Suppose $u \in C^{2}(\Omega), \Omega \subset \mathbb{R}^{n}$, is a solution to (6.5). Then $\Gamma=(x, \nabla u(x))$ is absolutely volume minimizing among all homologous $n$-surfaces in $\left(\mathbb{R}^{n} \times \mathbb{R}^{n}, g_{t}\right)$.

Using a change of variables, we may restate the following Bernstein-type results of Jörgens-Calabi-Pogorelov, Flanders, and Yuan.

Theorem. i) (Jörgens [7, Calabi [1, Pogorelov [13]) Suppose $u \in C^{2}\left(\mathbb{R}^{n}\right), D^{2} u \geq$ $-\cot t$, and $\Gamma=(x, \nabla u(x))$ is a maximal space-like surface in $\left(\mathbb{R}^{n} \times \mathbb{R}^{n}, g_{t}\right)$. Then $u(x)$ is a quadratic polynomial.

ii) (Flanders [3]) Suppose $u \in C^{2}\left(\mathbb{R}^{n}\right)$ is a convex solution to (6.4). Then $u(x)$ is a quadratic polynomial.

iii) (Yuan [14, [15]) There is a value $C_{t}$ such that if $u \in C^{2}\left(\mathbb{R}^{n}\right)$ is a solution to (6.5), with either

a) $D^{2} u \geq-\cot t$ or

b) $c>C_{t}+\frac{(n-2) \pi}{2}$, then $u(x)$ is a quadratic polynomial.

For $t \in\left[0, \frac{\pi}{2}\right]$, let $M_{t}=\left(\mathbb{R}^{n} \times \mathbb{R}^{n}, g_{t}\right)$, with $g_{t}$ as defined in (6.1). For $t<\frac{\pi}{4}, M_{t}$ is a pseudo-Euclidean space of index $n$. For $t>\frac{\pi}{4}, M_{t}$ is a Euclidean space. For $t=\frac{\pi}{4}, M_{t}$ carries a degenerate metric of rank $n$. In any case, an extremal (minimal or maximal) submanifold $\Sigma$ satisfies $H=0$ along $\Sigma$, where $H$ is the mean curvature vector along $\Sigma$.

Lemma 6.2. Suppose $u \in C^{3}(\Omega)$ and $\Gamma=(x, \nabla u(x))$ defines an extremal surface in $M_{t}, t \neq \pi / 4$. If $D^{2} u$ is diagonalized at a point $p=\left(x_{0}, \nabla u\left(x_{0}\right)\right)$, the mean curvature satisfies

$$
H=0 \stackrel{p}{=} \sum_{i, k}\left(\frac{u_{k i i}}{\sin t\left(1+\lambda_{i}^{2}\right)+2 \cos t \lambda_{i}} \partial_{y_{k}}\right)^{N},
$$

where ${ }^{N}$ denotes orthogonal projection onto $N(\Gamma)$, the normal bundle of $\Gamma$.

Proof. For an embedded submanifold of $\mathbb{R}^{n} \times \mathbb{R}^{n}$ given by

$$
f: \Omega \rightarrow \Gamma \hookrightarrow M_{t},
$$

we can compute the mean curvature vector $H$ by

$$
H=\left(g^{i j} f_{i j}\right)^{N},
$$

where $g$ is the induced metric on $\Gamma$, and $g^{i j}=\left(g^{-1}\right)_{i j}$. In this case,

$$
f(x)=\left(x_{1}, \ldots, x_{n}, u_{1}(x), \ldots, u_{n}(x)\right),
$$

so

$$
H=\left(g^{i j}\left(0,0, \ldots, 0, u_{1 i j}, \ldots, u_{n i j}\right)\right)^{N} .
$$

When $D^{2} u=\operatorname{diag}\left(\lambda_{1}, \ldots, \lambda_{n}\right)$,

$$
H=\sum_{i, k}\left(\frac{u_{k i i}}{\sin t\left(1+\lambda_{i}^{2}\right)+2 \cos t \lambda_{i}} \partial_{y_{k}}\right)^{N}=0,
$$


and $\left(\partial_{y_{k}}\right)^{N}$ is a linear combination of $\partial_{x_{k}}$ and $\partial_{y_{k}}$. For $k=1, \ldots, n$ the vectors $\left(\partial_{y_{k}}\right)^{N}$ are independent and form a basis for the normal space at $p$. It follows that

$$
\sum_{i} \frac{u_{k i i}}{\sin t\left(1+\lambda_{i}^{2}\right)+2 \cos t \lambda_{i}}=0
$$

for all $k$.

We now define the nonlinear operators $F^{t}\left(D^{2} u\right)$ by the equations (6.3) - (6.5).

At a point $p=\left(x_{0}, \nabla u\left(x_{0}\right)\right)$, we may diagonalize $D^{2} u$ and differentiate $F^{t}\left(D^{2} u\right)$. If the eigenvalues $\lambda_{i}$ are all distinct, then each is differentiable, and we have

$$
\frac{\partial}{\partial x_{k}} \lambda_{i} \stackrel{p}{=} \frac{\partial}{\partial x_{k}} u_{i i}=u_{i i k}
$$

and

$$
\begin{gathered}
\frac{\partial}{\partial x_{k}} F^{t}\left(D^{2} u(x)\right)=\sum_{i} \frac{1}{\sin t\left(1+\lambda_{i}^{2}\right)+2 \cos t \lambda_{i}} \frac{\partial}{\partial x_{k}} \lambda_{i} \\
\underline{\underline{p}} \sum_{i} \frac{u_{k i i}}{\sin t\left(1+\lambda_{i}^{2}\right)+2 \cos t \lambda_{i}}
\end{gathered}
$$

If the eigenvalues are not distinct, then one can use an eigenspace projection argument to verify that

$$
\frac{\partial}{\partial x_{k}} \sum_{i \in I_{j}} \lambda_{i} \stackrel{p}{=} \sum_{i \in I_{j}} u_{i i k}
$$

where $I_{j}=\left\{i \in 1, \ldots, n \mid \lambda_{i} \stackrel{p}{=} \lambda_{j}\right\}$, so that (6.7) holds in this case as well. It follows that the mean curvature $H$ vanishes if and only if $\nabla F^{t}\left(D^{2} u\right)=0$, that is, if and only if $F^{t}\left(D^{2} u\right)$ is constant.

For this family of nonlinear equations,

$$
\frac{\partial F^{t}}{\partial \lambda_{i}}=g^{i i}
$$

so we see that solutions are elliptic precisely when the Lagrangian submanifold is space-like. If we differentiate a second time with respect to $\lambda_{i}$, we see that the equation is convex when all $\lambda_{i}<-a$ and concave when all $\lambda_{i}>-a$. We note that as $t \rightarrow \frac{\pi}{4}$, equation (6.3) with $c=n \ln (a-b)$ becomes (6.4) with $c=\frac{n}{2}$. Equation (6.4) has been studied by Flanders [3], who obtained Bernstein Theorem ii).

6.1. Calibrations for $M_{t}$. Gradient graphs for solutions to (6.3) and (6.5) give rise to calibrated submanifolds of $M_{t}$, that are in fact isometric to calibrated submanifolds of $M_{0}$ and $M_{\frac{\pi}{2}}$. The calibrations for $M_{t}$ may be obtained by pulling back the calibrations on $M_{0}$ and $M_{\frac{\pi}{2}}$ via an isometry.

Throughout this section we will again be using the constants $a=\cot t$ and $b=\sqrt{\left|\cot ^{2} t-1\right|}$, for $t \in\left[0, \frac{\pi}{2}\right]$, as well as the constants defined by

$$
\begin{aligned}
\sigma & =\frac{\sqrt{\cos t+\sin t}+\sqrt{|\cos t-\sin t|}}{2}, \\
\tau & =\frac{\sqrt{\cos t+\sin t}-\sqrt{|\cos t-\sin t|}}{2} .
\end{aligned}
$$


We start with the pseudo-Euclidean metrics. For $t<\frac{\pi}{4}$, the map $\varphi_{t}: M_{t} \rightarrow M_{0}$, represented by the $2 n \times 2 n$ matrix

$$
\varphi_{t}=\left(\begin{array}{cc}
\sigma I & \tau I \\
\tau I & \sigma I
\end{array}\right)
$$

is an isometry (up to a constant factor). The Lagrangian condition is preserved under $\varphi_{t}$, so this isometry maps Lagrangian $n$-surfaces to Lagrangian $n$-surfaces. Pulling back the calibrations on $M_{0}$ described in section 2.2, $M_{t}$ becomes a calibrated manifold. The isometry gives an equivalence between the homogeneous space structure for the space-like Lagrangian planes of $M_{t}$ and the homogeneous space structure for the space-like Lagrangian planes of $M_{0}$ presented in section 4 .

Proof of Theorem 6.1. i). Suppose $u(x)$ is a solution to (6.3). Let $\Gamma=(x, \nabla u(x)) \subset$ $M_{t}$ be the graph of $\nabla u$. Our goal is to show that $\Gamma$ is isometric to a special Lagrangian graph $\hat{\Gamma} \subset M_{0}$. Take $\hat{\Gamma}=\varphi_{t}(\Gamma) \subset M_{0}$. At a point $p=\left(x_{0}, \nabla\left(x_{0}\right)\right) \in \Gamma$, the tangent space of $\Gamma$ can be described by the span of the vectors

$$
\partial_{i}=\partial_{x_{i}}+\sum_{j} u_{i j} \partial_{y_{j}}, \quad i=1, \ldots, n .
$$

Take $D^{2} u$ to be diagonalized at $p$, and push forward. The tangent space $T_{\varphi_{t}(p)} \hat{\Gamma}=$ $\left(\varphi_{t}\right)_{*}\left(T_{p} \Gamma\right)$ is the span of

$$
\hat{\partial}_{i}=\left(\varphi_{t}\right)_{*} \partial_{i}=\left(\sigma+\tau \lambda_{i}\right) \partial_{x_{i}}+\left(\tau+\sigma \lambda_{i}\right) \partial_{y_{i}} .
$$

The space-like condition on $\Gamma$ imposes restrictions on the values of $\lambda_{i}$, particularly, $\lambda_{i} \neq-\sigma / \tau$, so we may multiply each $\hat{\partial}_{i}$ by $1 /\left(\sigma+\tau \lambda_{i}\right)$ and see that $T_{\varphi_{t}(p)} \hat{\Gamma}$ is spanned by

$$
\hat{\partial}_{x_{i}}+\frac{\tau+\sigma \lambda_{i}}{\sigma+\tau \lambda_{i}} \hat{\partial}_{y_{i}}
$$

The image $\hat{\Gamma}$ is a Lagrangian submanifold of $M_{0}$, so it arises locally as the gradient graph $\hat{\Gamma}=(\hat{x}, \nabla \hat{u}(\hat{x}))$. From the above expression, the eigenvalues of $D^{2} \hat{u}$ are given by

$$
\hat{\lambda}_{i}=\frac{\tau+\sigma \lambda_{i}}{\sigma+\tau \lambda_{i}}=\frac{\left(\lambda_{i}+\tau / \sigma\right)}{\left(\lambda_{i}+\sigma / \tau\right)} \frac{(\sigma)}{(\tau)} .
$$

The $\lambda_{i}$ 's satisfy (6.3), so noting that $a+b=1 /(a-b)=\sigma / \tau$, we have

$$
\prod_{i=1}^{n} \frac{\lambda_{i}+\tau / \sigma}{\lambda_{i}+\sigma / \tau}=e^{c}>0
$$

and we may conclude that $\hat{u}(\hat{x})$ satisfies

$$
\prod_{i=1}^{n} \hat{\lambda}_{i}=\left[\frac{\sigma}{\tau}\right]^{n} e^{c}>0
$$

that is, $\hat{u}(\hat{x})$ satisfies the Monge-Ampère equation (1.1). It follows that $\hat{\Gamma}$ is a calibrated submanifold. The property of being calibrated is local and is preserved under isometries of the ambient manifolds, so $\Gamma$ is calibrated. Theorem 6.1 $i$ ) then follows by the same reasoning as in the proof of Theorem 3.2 
The function $\hat{u}$ obtained above is only local. In order to study solutions to 6.3. further, in particular to obtain uniqueness for Theorem 6.1 i) and the Bernsteintype result, we transform $u$ into a solution to (1.1) which describes $\hat{\Gamma}$ globally, when possible.

The isometry $\varphi_{t}$ acts via

$$
\varphi_{t}(x, y)=(\sigma x+\tau y, \tau x+\sigma y)=(p(x, y), q(x, y)),
$$

so the image $\hat{\Gamma}=\varphi_{t}(\Gamma)$ lies in the set $p(\Gamma) \times q(\Gamma) \subset \mathbb{R}^{n} \times \mathbb{R}^{n}$. As $\Gamma$ is parameterized by $\Omega$, the isometry $\Gamma \rightarrow \hat{\Gamma}$ locally amounts to a change of coordinates $\Omega \rightarrow p(\Gamma)$, wherever $D p$ is invertible. If $\left.p\right|_{\Gamma}$ is a bijection, then $\hat{\Gamma}$ is globally parameterized by $p(\Gamma)$. In the case where $\left.p\right|_{\Gamma}$ is a bijection, $\hat{\Gamma}$ is a graph over $p(\Gamma)$ of the function

$$
r=q \circ p^{-1}: p(\Gamma) \rightarrow \mathbb{R}^{n} .
$$

Using $\Omega$ as coordinates for $\Gamma$

$$
\begin{aligned}
& p(x)=\sigma x+\tau \nabla u(x), \\
& q(x)=\tau x+\sigma \nabla u(x) ;
\end{aligned}
$$

hence

$$
\begin{aligned}
& D p=\sigma I+\tau D^{2} u, \\
& D q=\tau I+\sigma D^{2} u,
\end{aligned}
$$

so $p$ is locally invertible if and only if no eigenvalues of $D^{2} u$ attain the value $-\sigma / \tau=$ $-(a+b)$, which is precluded by the space-like condition. Diagonalizing $D^{2} u$, we see that $D r$ is symmetric, so $r=\nabla \hat{u}$ for some $\hat{u}$, and the eigenvalues of $D^{2} \hat{u}$ become

$$
\hat{\lambda}_{i}=\frac{\tau+\sigma \lambda_{i}}{\sigma+\tau \lambda_{i}}
$$

as in (6.8). Now if $D^{2} u \geq-a>-(a+b)$, then $D p>0$ and $p$ is injective on $\Omega$. The function $\hat{u}$ is then a solution to the Monge-Ampère equation defined on all of $p(\Gamma)$. Further, the inequality is uniform, $D p \geq \epsilon>0$. Thus if $\Omega=\mathbb{R}^{n}, p$ is a bijection on $\mathbb{R}^{n}$, so $\hat{u}$ will then be a convex solution to (1.1) on all of $\mathbb{R}^{n}$. Bernstein Theorem i) follows from the famous result

Theorem (Jörgens [7, Calabi [1], Pogorelov [13]). Any convex solution to (1.1) on all of $\mathbb{R}^{n}$ is a quadratic polynomial.

Using a recent, more general result, we see that we may drop the restriction that $D^{2} \geq-a$.

Theorem (Jost-Xin, 8 Theorem 4.2). Let $M$ be a space-like extremal m-surface in $\mathbb{R}_{n}^{n+m}$. If $M$ is closed with respect to the Euclidean topology, then $M$ must be a linear subspace.

Uniqueness. From the above discussion, if we assume that $D^{2} u \geq-a$, the surface $\Gamma$ can be described globally as the gradient graph of a solution to the Monge-Ampère equation. In this case, the fact that $\Gamma$ is the unique surface with this volume follows from Theorem 3.2. The proof of Theorem 3.2 relied heavily on the fact that the space-like condition forced the projection $\Gamma \rightarrow \mathbb{R}_{x}^{n}$ to be an open map. This is not the case for gradient graphs of solutions to equation (6.3), so the corresponding proof of uniqueness will not work. We do not have a proof of uniqueness for equation (6.3) with no restrictions on $D^{2} u$, nor for the analogous 
result for solutions to (6.5). As in ([5], Theorem 5.8), we do have uniqueness for solutions to (6.3) and (6.5) whenever the boundary data is analytic, as an application of the Cauchy-Kowaleswki Theorem.

Proof of Theorem 6.1, ii) Let $P: \mathbb{R}^{n} \times \mathbb{R}^{n} \rightarrow \mathbb{R}^{n}$ be the map

$$
(x, y) \mapsto \frac{x+y}{2}
$$

Given the degenerate metric $g_{\frac{\pi}{4}}$ on $\mathbb{R}^{n} \times \mathbb{R}^{n}$, this map is an "isometry" in the sense that $g_{\frac{\pi}{4}}=P^{*} \delta_{0}$. The graph $\Gamma=(x, F(x))$ is isometric to $P(\Gamma)$ for any $F(x)$ such that $D F$ avoids the eigenvalue $\lambda_{i}=-1$. Let $\Sigma$ be any space-like surface with boundary $\partial \Sigma=\Gamma \cap\{x \in \Omega\}$. Then if $\omega=x_{n} d x_{1} \wedge \ldots \wedge d x_{n-1}$, so that $d w=d V o l$ on $\mathbb{R}^{n}$,

$$
\begin{aligned}
\operatorname{Vol}(\Sigma)= & \int_{\Sigma} d V o l=\int_{\Sigma} P^{*} d V o l=\int_{\Sigma} P^{*} d w \\
& =\int_{\Sigma} d P^{*} w=\int_{\partial \Sigma} P^{*} w
\end{aligned}
$$

which depends only on the boundary $\partial \Gamma=\partial \Sigma$.

Proof of Theorem 6.1, iii) As in the pseudo-Euclidean case, for $t>\frac{\pi}{4}$, the map $\varphi_{t}: M_{t} \rightarrow M_{\frac{\pi}{2}}$ is an isometry. For a given $n$-plane $\xi$,

$$
\begin{aligned}
& \operatorname{Vol}_{g_{t}}^{2}(\xi)=\operatorname{Vol}_{\delta_{0}}^{2}\left(\varphi_{t} \xi\right) \geq \operatorname{Vol}\left(\varphi_{t} \xi \wedge J \varphi_{t} \xi\right) \\
= & \operatorname{det}_{\mathbb{R}} A=\left\|\operatorname{det}_{\mathbb{C}} A\right\|^{2}=\alpha^{2}(\xi)+\beta^{2}(\xi) \geq \alpha^{2}(\xi),
\end{aligned}
$$

where $A \in M(n, \mathbb{C}) \subset M(2 n, \mathbb{R})$ is the map sending $\partial_{x_{i}} \mapsto \varphi v_{i}$, extended by complex linearity as before. By Hadamard's Inequality, we have equality if and only if $\xi$ is Lagrangian and $\beta(\xi)=0$.

If $\xi$ is Lagrangian, then $\varphi_{t} v_{1}, \ldots, \varphi_{t} v_{n}$ is an orthonormal basis for $\mathbb{C}^{n}$, so $A \in$ $U(n)$. Using the map

$$
\Theta: L \approx U(n) / S O(n) \stackrel{\operatorname{detc}_{\mathrm{c}}}{\rightarrow} S^{1},
$$

the special Lagrangian equations (6.5) can be deduced from

$$
\arg \operatorname{det}\left(\sigma+\tau D^{2} u+\sqrt{-1}\left(\tau+\sigma D^{2} u\right)\right)=\sum \arctan \left(\frac{\tau+\sigma \lambda_{i}}{\sigma+\tau \lambda_{i}}\right)=c .
$$

It follows that any gradient graph $\Gamma=(x, \nabla u(x))$ for $u(x)$ satisfying this equation is calibrated and is therefore an absolutely volume minimizing submanifold.

By differentiating with respect to $\lambda$, one can verify the identity

$$
\sum \arctan \frac{\lambda_{i}+a}{b}+C_{t}=\sum \arctan \left(\frac{\tau+\sigma \lambda_{i}}{\sigma+\tau \lambda_{i}}\right),
$$

where

$$
C_{t}=\arctan \left(\frac{\tau}{\sigma}\right)-\arctan \frac{a}{b} .
$$

It follows that a solution to (6.5) also satisfies

$$
\sum \arctan \frac{\lambda_{i}+a}{b}=c-C_{t}
$$

and the function

$$
v(x)=\frac{u(x)}{b}+\frac{a}{2 b}|x|^{2}
$$


is a solution to (1.2). If $v(x)$ is convex, which is the case if $D^{2} u \geq-a$, we may conclude that $v(x)$ is a quadratic polynomial by applying the following result.

Theorem (Yuan [14). Suppose $u \in C^{2}\left(\mathbb{R}^{n}\right)$ is a convex solution to (1.2). Then $u(x)$ is a quadratic polynomial.

Similarly, any solution to (6.5) with $c>(n-2) \pi / 2+C_{t}$ is a quadratic polynomial, by the theorem

Theorem (Yuan [15]). Suppose $u \in C^{2}\left(\mathbb{R}^{n}\right)$ is a solution to (1.2) with $c>\frac{(n-2) \pi}{2}$. Then $u(x)$ is a quadratic polynomial.

\section{EXAMPLE}

The equations (6.3)-(6.5) can be manipulated to take the form

$$
f\left(\lambda_{1}, \ldots, \lambda_{n}\right)=0,
$$

where $f$ is a polynomial of degree no higher than $n$. For equation (6.3) with $c=0$, the polynomial $f$ has degree $n-1$. When $n=2$, (6.3) with $c=0$ becomes a linear equation, taking the form $\Delta u=-2 a$. We exploit this degeneracy in order to write an explicit solution to (6.3).

Fix some $t \in\left(0, \frac{\pi}{4}\right)$, and define $u\left(x_{1}, x_{2}\right)$ on $\Omega=\left\{x_{1}>0 \subset \mathbb{R}^{2}\right\}$

$$
u\left(x_{1}, x_{2}\right)=-\frac{a}{2}\left(x_{1}^{2}+x_{2}^{2}\right)+k e^{x_{1}} \cos x_{2},
$$

for $k$ some large constant, and $a=\cot t$. The eigenvalues of $D^{2} u$ are

$$
\begin{aligned}
& \lambda_{1}=-a-k e^{x_{1}}, \\
& \lambda_{2}=-a+k e^{x_{1}} .
\end{aligned}
$$

Clearly, $u$ is a solution to (6.3) with $c=0$, that is, $\Delta u=-2 a$, and one can check that the resulting surface $\Gamma=(x, \nabla u(x))$ is space-like in $\left(\mathbb{R}^{2} \times \mathbb{R}^{2}, g_{t}\right)$. We transform this to a maximal submanifold of $\left(\mathbb{R}^{n} \times \mathbb{R}^{n}, g_{0}\right)$, using $\varphi_{t}$. This isometry acts via

$$
\varphi_{t}(x, y)=(\sigma x+\tau y, \tau x+\sigma y)
$$

so the image $\varphi_{t}(\Gamma)=\hat{\Gamma} \subset\left(\mathbb{R}^{4}, g_{0}\right)$ lies in $p(\Gamma) \times \mathbb{R}^{2}$, where $p(x, y)=\sigma x+\tau y$. Parameterizing $\Gamma$ by $\Omega$, the map $\left.p\right|_{\Gamma}$ takes the form

$$
\begin{gathered}
p\left(x_{1}, x_{2}\right)=\sigma \cdot\left(x_{1}, x_{2}\right)+\tau \cdot \nabla u\left(x_{1}, x_{2}\right) \\
\text { or } p(z)=(\sigma-\tau a) z+\tau k e^{\bar{z}},
\end{gathered}
$$

where $z=x_{1}+i x_{2}$. For large $k$, tracing the path of the image of a vertical line shows that $p$ cannot be injective on $\Gamma$, so $\hat{\Gamma}$ will not be described globally as a graph over $p(\Gamma)$. This surface $\hat{\Gamma}$ is a maximal Lagrangian surface which is globally described as a solution to (6.3) and locally described as a solution to (1.1), but which is not globally described as a solution to (1.1). This surface is not complete, as we have restricted the domain to a half-plane, but by the result of Jost and Xin ( 8 , Theorem 4.1) one would not expect to find a complete surface with this property.

\section{ACKNOWLEDGMENT}

The author is happy to thank his thesis advisor, Yu Yuan, for suggesting this problem, and for the many fruitful discussions about this and other problems. 


\section{REFERENCES}

1. Eugenio Calabi, Improper affine hyperspheres of convex type and a generalization of a theorem by K. Jörgens, Michigan Math. J. 5 (1958), 105-126. MR0106487(21:5219)

2. Vicente Cortés, Christoph Mayer, Thomas Mohaupt, and Frank Saueressig, Special geometry of Euclidean supersymmetry. I. Vector multiplets, J. High Energy Phys. (2004), no. 3, 028, 73 pp. (electronic). MR.2061551(2005c:53055)

3. Harley Flanders, On certain functions with positive definite Hessian, Ann. of Math. (2) 71 (1960), 153-156. MR0145025 (26:2562)

4. David Gilbarg and Neil S. Trudinger, Elliptic partial differential equations of second order, second ed., Grundlehren der Mathematischen Wissenschaften [Fundamental Principles of Mathematical Sciences], vol. 224, Springer-Verlag, Berlin, 1983. MR.737190 (86c:35035)

5. Reese Harvey and H. Blaine Lawson, Jr., Calibrated geometries, Acta Math. 148 (1982), 47-157. MR666108 (85i:53058)

6. Nigel J. Hitchin, The moduli space of special Lagrangian submanifolds, Ann. Scuola Norm. Sup. Pisa Cl. Sci. (4) 25 (1997), no. 3-4, 503-515 (1998). Dedicated to Ennio De Giorgi. MR $1655530(2000 \mathrm{c}: 32075)$

7. Konrad Jörgens, Uber die Lösungen der Differentialgleichung $r t-s^{2}=1$, Math. Ann. 127 (1954), 130-134. MR0062326 (15:961e)

8. Jürgen Jost and Yuan-Long Xin, Bernstein type theorems for higher codimension, Calc. Var. Partial Differential Equations 9 (1999), no. 4, 277-296. MR.1731468(2001e:53010)

9. Young-Heon Kim and Robert J. McCann, Continuity, curvature, and the general covariance of optimal transportation, Journal of the European Mathematical Society (To Appear).

10. Young-Heon Kim, Robert J. McCann, and Micah Warren, Pseudo-Riemannian geometry calibrates optimal transportation, Preprint.

11. Jack Mealy, Volume maximization in semi-Riemannian manifolds, Indiana Univ. Math. J. 40 (1991), no. 3, 793-814. MR1129330 (92k:53123)

12. Robert C. McLean, Deformations of calibrated submanifolds, Comm. Anal. Geom. 6 (1998), no. 4, 705-747. MR 1664890 (99j:53083)

13. A. V. Pogorelov, On the improper convex affine hyperspheres, Geometriae Dedicata 1 (1972), no. 1, 33-46. MR0319126 (47:7672)

14. Yu Yuan, A Bernstein problem for special Lagrangian equations, Invent. Math. 150 (2002), no. 1, 117-125. MR1930884 (2003k:53060)

15. _ Global solutions to special Lagrangian equations, Proc. Amer. Math. Soc. 134 (2006), no. $5,1355-1358$.

Department of Mathematics, University of Washington, Box 354350, Seattle, WashINGTON 98195-4350

Current address: Department of Mathematics, Fine Hall, Princeton University, Washington Road, Princeton, New Jersey 08544-1000

E-mail address: mww@princeton.edu 